\title{
Midkine and syndecan-1 levels correlate with the progression of malignant gastric cardiac adenocarcinoma
}

\author{
XIU-FENG HU, JUN YAO, SHE-GAN GAO, YAN-TONG YANG, XIU-QING PENG and XIAO-SHAN FENG
}

Department of Oncology, Cancer Research Institute,

The First Affiliated Hospital of Henan University of Science and Technology,

Luoyang, Henan 471003, P.R. China

Received July 19, 2013; Accepted March 4, 2014

DOI: $10.3892 / \mathrm{mmr} .2014 .2369$

\begin{abstract}
The present study aimed to determine whether the expression levels of midkine (MK) and syndecan-1 correlate with the malignant progression and poor prognosis of gastric cardiac adenocarcinoma (GCA). GCA tissue samples $(n=72)$ were obtained from the Department of Pathology of the First Affiliated Hospital of Henan University of Science and Technology (Luoyang, China). The paraffin-embedded samples had been surgically resected and pathologically diagnosed between 2007 and 2009. Normal gastric cardiac biopsy specimens $(n=40)$ were also collected as the control. The expression levels of MK and syndecan-1 were assessed by immunohistochemistry using the high-sensitivity streptavidin-peroxidase method. Statistical analysis was performed on the data obtained using the SPSS 17.0 statistics package. MK expression was detected in $76.4 \%$ of GCA samples and $5 \%$ of normal gastric cardiac mucosa specimens. A significant positive correlation was observed between the expression levels of MK and the infiltrative depth of the tumor, the presence of lymph node metastasis and the prognosis of the patients $(\mathrm{P}<0.05)$. Syndecan-1 expression was detected in $38.9 \%$ of GCA samples and $100 \%$ of normal gastric cardiac mucosa samples. The expression levels of syndecan-1 were negatively correlated with the grade of differentiation, serosal membrane invasion, lymph node metastasis and the patient's prognosis $(\mathrm{P}<0.05)$. Notably, the expression levels of MK and syndecan-1 were inversely correlated $(\mathrm{r}=-0.352, \mathrm{P}<0.01)$ in the GCA tissue samples. These results suggest that high expression levels of MK in GCA tissues may indicate a differentiation stage that is characteristic of malignancy, a late clinical stage and a poor prognosis, whereas increased syndecan-1 levels may
\end{abstract}

Correspondence to: Professor Xiao-Shan Feng, Department of Oncology, Cancer Research Institute, The First Affiliated Hospital of Henan University of Science and Technology, 24 Jinghua Road, Luoyang, Henan 471003, P.R. China

E-mail: samfeng2013@hotmail.com

Key words: gastric cardiac adenocarcinoma, midkine, syndecan-1, immunohistochemistry, prognosis indicate a high degree of differentiation, an early clinical stage and a favorable prognosis. MK and syndecan-1 may serve as important biomarkers for monitoring the development and progression of GCA.

\section{Introduction}

Gastric cardiac adenocarcinomas (GCAs) are derived from the most proximal region of the stomach immediately adjoining the esophagus. GCA is one of the most common forms of malignant cancer in China. Epidemiological studies have revealed that the incidence of GCA has been increasing every year, and is rapidly becoming one of the leading causes of malignant tumors worldwide (1-3). Although there has been great progress in the diagnosis and treatment of GCA, the five-year survival rate remains at $<24 \%$. Therefore, there is an urgent requirement for the development of functional biomarkers for the early diagnosis of GCA and improved survival rates.

Midkine (MK) was originally identified as a retinoic acid-inducible gene in murine embryonic carcinoma cells. MK is known to be involved in early embryogenesis. In adults, low levels of MK are detectable in kidney tissue (4). However, MK expression is frequently upregulated in numerous solid tumors, including gastric cancer $(5,6)$. Deregulation of MK expression is associated with tumor invasion and a poor prognosis (7), indicating that MK may be involved in tumorigenesis.

MK is involved in the regulation of a large number of cellular activities. MK binds heparin to promote proliferation, survival and migration by activating cell growth signaling pathways. The interaction of MK with anaplastic lymphoma kinase (ALK) leads to the activation of the phosphoinositide-3 kinase/Akt and mitogen-activated protein kinase pathways. MK induces neurite outgrowth and migration by interacting with $\beta 1$-integrin (8). In addition, MK tightly binds proteoglycans (PGs), including syndecan-1 [also known as cluster of differentiation (CD)138] (9). Syndecan-1 has a role in cell growth and differentiation by interacting with several growth factors through its extracellular heparan sulfate (HS) glycosaminoglycan chains (10). The differentiation and morphogenesis of vertebrate embryos depends on the interaction of syndecan-1 with MK (9). Syndecan-1 is also involved in cell adhesion 
and migration by controlling cell-cell and cell-extracellular matrix interactions (11). The correlation between syndecan-1 and tumor development has been a controversial subject. It has been reported that the loss of syndecan-1 facilitates the migration of metastatic tumor cells, and is correlated with a poor prognosis in patients with head and neck, non-small cell lung and hepatocellular carcinomas $(12,13)$. Furthermore, another study has indicated that the overexpression of syndecan-1 is correlated with a poor outcome in patients with cancer (14). However, further studies are required to confirm the role of syndecan-1 in tumor development and progression.

A concomitant increase in MK expression and a decrease in syndecan-1 expression in certain cancer types prompted the present study to examine the expression levels of $\mathrm{MK}$ and syndecan-1 in the same GCA tissue samples, and the exploration of the roles of MK and syndecan-1 in GCA development, invasion and metastasis. The present study assessed the expression levels of MK and syndecan-1 in 72 surgically obtained GCA tissue samples and 40 normal gastric cardia tissue samples, and assessed the correlation between MK and syndecan-1 expression and the clinicopathological features of GCA.

\section{Materials and methods}

Patients and tissue samples. A total of 72 paraffin-embedded GCA tissue samples were obtained from the Department of Pathology of the First Affiliated Hospital of Henan University of Science and Technology (Luoyang, China). The paraffin-embedded samples had been surgically resected and pathologically diagnosed between 2007 and 2009. The research protocol and the consent forms were approved by the Institutional Committee for the Protection of Human Subjects. The 72 GCA samples had been extracted from 56 male and 16 female patients. The average age of the patients was $55.4 \pm 7.56$ years. A total of 40 samples of normal tissue were obtained from 28 males and 12 females, with an average age of $55.3 \pm 8.3$ years. None of the patients received radiotherapy or chemotherapy treatment prior to the surgery.

Reagents and antibodies. Rabbit anti-human MK polyclonal antibody was obtained from Santa Cruz Biotechnology, Inc., (bs-1849R; Dallas, TX, USA) and mouse anti-syndecan-1 monoclonal antibody was obtained from Beijing Zhongshan Jinqiao Biotechnology (ZM-0459; Beijing, China). Anti-rabbit and anti-rat streptavidin-peroxidase (SP) high-sensitivity kits were purchased from Fuzhou Maixin Biotechnology (KIT-9710; Fujian, China).

Immunohistochemistry and criteria for analysis of results. Three $4-\mu \mathrm{m}$ thick slices cut from the formalin-fixed, paraffin-embedded tissue blocks were used for immunohistochemical staining. Known positive tissue sections served as positive controls. All immunohistochemical data were evaluated by two pathologists separately. MK and syndecan-1 staining was visible on the cell membrane and/or in the cytoplasm. The presence of clear yellow-brown granules in the cytoplasm or on the cell membrane was considered as positive staining for MK and syndecan-1. Semi-quantitative evaluation was performed, with scores as follows: $0,<10 \%$ positive cells $(-) ; 1,10-49 \%$ positive cells $(+) ; 2,50-75 \%$ positive cells $(++)$; and $3,>75 \%$ positive cells $(+++)$ displaying cytoplasmic and membrane-associated immunoreactivity. Scores of 1,2 and 3 were interpreted as positive (+) staining in all tissues.

Statistical analysis. Statistical analysis was performed using the SPSS 17.0 statistical software package (SPSS, Inc., Chicago, IL, USA). The $\chi^{2}$ or Fisher's exact probability tests were applied for the analysis of qualitative variables, and the Spearman's rank correlation coefficient was used to analyze the correlation between $\mathrm{MK}$ and syndecan- 1 . $\mathrm{P}<0.05$ was used to indicate a statistically significant difference. Overall survival curves were generated using the Kaplan-Meier method, and the differences between the groups were analyzed using the log-rank test.

\section{Results}

Expression levels of $M K$ and syndecan-1 in GCA and atypical hyperplasia tissues. Immunohistochemical staining showed that MK and syndecan-1 were expressed on the cell membranes and/or in the cytoplasm. Among the GCA samples, 76.4\% (55/72) exhibited positive staining for MK (Fig. 1A), whereas only $5 \%(2 / 40)$ of the normal gastric cardiac specimens showed positive staining for MK $(\mathrm{P}<0.05)$ (Fig. 1B). Positive staining for syndecan-1 was observed in $100 \%$ (40/40) of the normal gastric cardiac specimens (Fig. 2A), whereas $38.9 \%(28 / 72)$ of GCA tissue specimens showed syndecan-1 staining $(\mathrm{P}<0.05)$ (Fig. 2B). Significant differences were observed between normal cardiac mucosa and GCA tissue samples for MK and syndecan-1 levels $(\mathrm{P}<0.05)$ (Table I). These data demonstrate that the expression levels of MK had increased, whereas those of syndecan-1 had decreased in progressive GCA, indicating that MK and syndecan-1 have significant roles in the tumorigenesis of GCA.

Association between the clinicopathological features of GCA and the expression levels of MK or syndecan-1. MK expression levels were positively correlated with metastases of the lymph node $\left(\chi^{2}, 8.50 ; \mathrm{P}<0.05\right)$ and stromal invasion $\left(\chi^{2}, 5.073 ; \mathrm{P}<0.01\right)$. MK expression was not associated with age, gender, tumor size or the degree of histological differentiation of GCA $(\mathrm{P}>0.05)$ (Table II). Logistic regression analysis showed that the MK levels were closely associated with metastases to the lymph nodes $(\mathrm{P}=0.007)$ and the depth of serosal membrane invasion $(\mathrm{P}=0.018)$ (Table III). Univariate analysis showed that the expression levels of syndecan-1 were not correlated with age, gender or tumor size $(\mathrm{P}>0.05)$, but that they were negatively correlated with the degree of histological differentiation of GCA $\left(\chi^{2}, 6.768\right.$; $\mathrm{P}<0.05)$, stromal invasion $\left(\chi^{2}, 7.182 ; \mathrm{P}<0.01\right)$ and metastases of the lymph nodes $\left(\chi^{2}, 10.979 ; \mathrm{P}<0.01\right)$ (Table II). Similarly, logistic regression analysis demonstrated that syndecan-1 levels were not associated with metastases to the lymph nodes $(\mathrm{P}=0.271)$ or the degree of differentiation $(\mathrm{P}=0.307)$, but that they were closely associated with the depth of serosal membrane invasion ( $\mathrm{P}=0.021)$ (Table IV). Therefore, MK expression levels were correlated with serosal membrane invasion and lymph node metastases, while syndecan-1 
Table I. Expression levels of MK and syndecan-1 in various gastric cardiac mucosal tissue samples. ${ }^{\mathrm{a}}$

\begin{tabular}{|c|c|c|c|c|c|c|c|c|}
\hline \multirow[b]{2}{*}{ Group } & \multicolumn{2}{|c|}{ MK, n (\%) } & \multirow[b]{2}{*}{$\chi^{2}$} & \multirow[b]{2}{*}{ P-value } & \multicolumn{2}{|c|}{ Syndecan-1, n (\%) } & \multirow[b]{2}{*}{$\chi^{2}$} & \multirow[b]{2}{*}{ P-value } \\
\hline & Negative & Positive & & & Negative & Positive & & \\
\hline Normal cardiac mucosa $(n=40)$ & $38(95.0)$ & $2(5.0)$ & 52.437 & $<0.01$ & $0(0.0)$ & $40(100.0)$ & 40.261 & $<0.01$ \\
\hline $\operatorname{GCA}(n=72)$ & $17(24.6)$ & $55(76.4)$ & & & $44(61.1)$ & $28(38.9)$ & & \\
\hline
\end{tabular}

${ }^{\mathrm{a}} \mathrm{An}$ increased number of GCA tissue samples were positive for MK expression compared with the normal gastric cardiac mucosa tissues $(\mathrm{P}<0.01)$. An increased number of GCA tissue samples were negative for syndecan-1 expression compared with the normal gastric cardiac mucosa samples. MK, midkine; GCA, gastric cardiac adenocarcinoma.

Table II. Association between clinicopathological features of GCA and expression of MK or syndecan-1.

\begin{tabular}{|c|c|c|c|c|c|c|}
\hline \multirow[b]{2}{*}{ Factors } & \multicolumn{3}{|c|}{ MK } & \multicolumn{3}{|c|}{ Syndecan-1 } \\
\hline & Positive, $\mathrm{n}$ & $\%$ & P-value & Positive, $\mathrm{n}$ & $\%$ & P-value \\
\hline \multicolumn{7}{|l|}{ Gender } \\
\hline Male $(\mathrm{n}=56)$ & 45 & 80.4 & 0.152 & 22 & 39.3 & 0.897 \\
\hline Female $(n=16)$ & 10 & 62.5 & & 6 & 37.5 & \\
\hline \multicolumn{7}{|l|}{ Age (years) } \\
\hline$<60(\mathrm{n}=34)$ & 28 & 82.4 & 0.256 & 16 & 47.1 & 0.554 \\
\hline$\geq 60(\mathrm{n}=38)$ & 27 & 71.1 & & 12 & 31.6 & \\
\hline \multicolumn{7}{|l|}{ Depth of invasion } \\
\hline No stromal invasion $(n=25)$ & 15 & 60.0 & 0.017 & 15 & 60.0 & 0.007 \\
\hline Invaded stroma $(\mathrm{n}=47)$ & 40 & 85.1 & & 13 & 27.7 & \\
\hline \multicolumn{7}{|l|}{ Degree of differentiation } \\
\hline $\operatorname{High}(\mathrm{n}=11)$ & 9 & 81.8 & 0.894 & 8 & 72.7 & 0.034 \\
\hline Moderate $(n=45)$ & 34 & 75.6 & & 16 & 35.6 & \\
\hline Low $(n=16)$ & 12 & 75.0 & & 4 & 25.0 & \\
\hline \multicolumn{7}{|l|}{ Lymph node metastasis } \\
\hline Positive $(n=43)$ & 38 & 88.4 & 0.004 & 10 & 23.3 & 0.001 \\
\hline Negative $(n=29)$ & 17 & 58.6 & & 18 & 62.1 & \\
\hline
\end{tabular}

MK, midkine; GCA, gastric cardiac adenocarcinoma.

Table III. Logistic regression analysis for the association between MK levels and lymph node metastasis or serosal membrane invasion.

\begin{tabular}{lccccccrr}
\hline Parameter & \multirow{2}{*}{ S } & & & & & & \multicolumn{2}{c}{$95 \%$ CI for Exp (B) } \\
\hline Lymph node metastasis & -1.723 & 0.641 & 7.232 & 1 & 0.007 & 0.178 & 0.051 & 0.627 \\
Serosal membrane invasion & 1.483 & 0.629 & 5.550 & 1 & 0.018 & 4.404 & 1.283 & 15.119 \\
\hline
\end{tabular}

CI, confidence interval; df, degrees of freedom; SE, standard error; MK, midkine.

expression levels were correlated with serosal membrane invasion only.

Expression levels of $M K$ and syndecan-1 are inversely correlated. The correlation between the expression levels of MK and syndecan-1 was assessed for the 72 cases of GCA by Spearman's rank correlation analysis. It was revealed that the expression levels of MK were negatively correlated with those of syndecan-1. Increases in the levels of MK led to concomitant decreases in the levels of syndecan-1 $(\mathrm{r}=-0.352 ; \mathrm{P}<0.01)($ Table $\mathrm{V})$. 

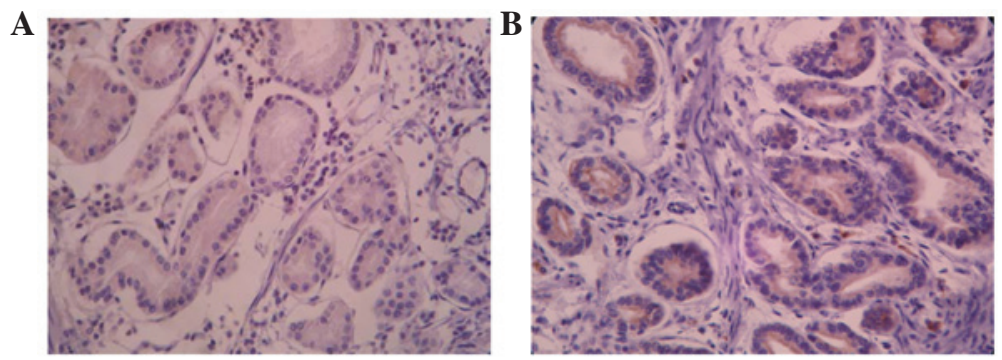

Figure 1. Immunohistochemical staining of MK protein in (A) normal gastric tissue and (B) GCA tissue. Paraffin-embedded gastric serial sections were stained with MK antibodies. Low expression of MK was detected in normal tissue compared with GCA tissue. The staining showed that MK was mainly localized on the cell membranes and/or in the cell cytoplasm with yellow-brown granules in the nuclei (magnification, x200). MK, midkine; GCA, gastric cardiac adenocarcinoma.
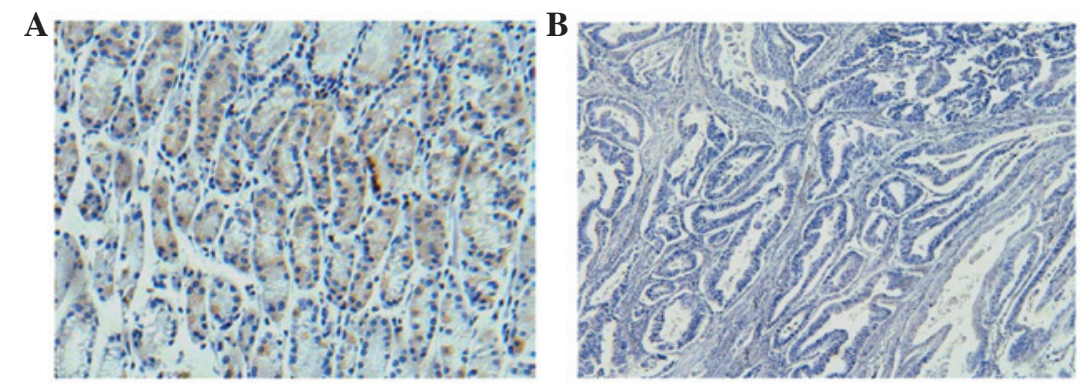

Figure 2. Immunohistochemical staining of syndecan-1 protein in (A) normal gastric tissue and (B) GCA tissue (magnification, x100). Positive staining for syndecan-1 was observed in the normal gastric tissue, whereas a lack of staining or low expression of syndecan-1 was detected in the GCA tissue. GCA, gastric cardiac adenocarcinoma.

A

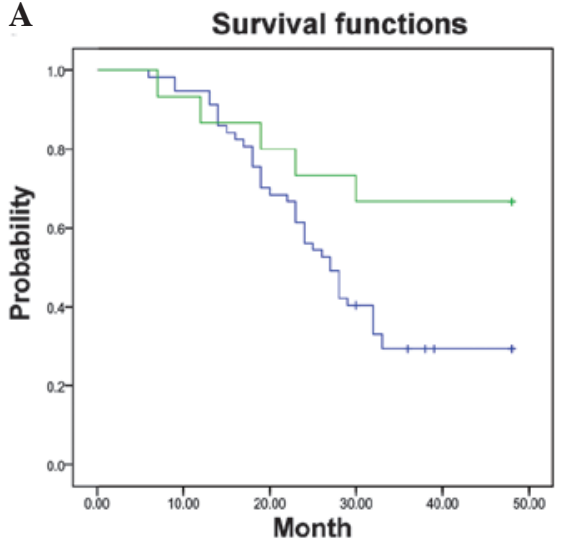

B

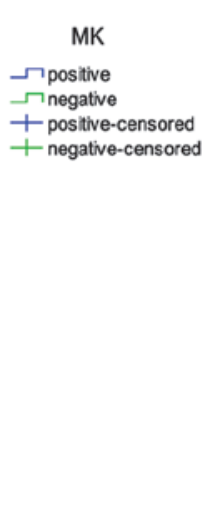

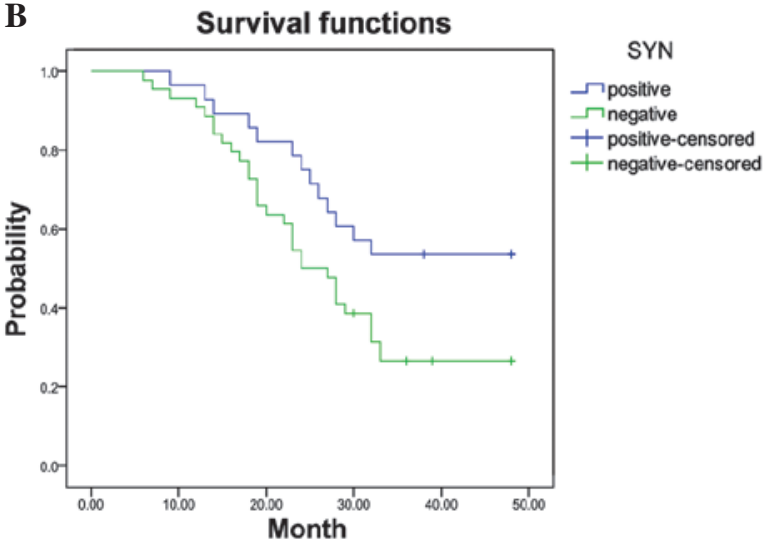

Figure 3. Association between prognosis of GCA and expression of (A) MK or (B) syndecan-1. Survival curves for 72 patients with GCA, as associated with the expression of (A) MK and (B) syndecan-1 in tumor tissue. The median survival time and three-year survival rates were lower in patients who were positive for MK and/or negative for syndecan-1 expression compared with patients who were negative for MK and/or positive for syndecan-1 expression. MK, midkine; GCA, gastric cardiac adenocarcinoma; SYN, syndecan-1.

Association between the prognosis of GCA and the expression levels of $M K$ or syndecan-1. Among the 72 cases of GCA, the median survival time for patients with increased MK expression was 27 months [95\% confidence interval (CI), 23.3-30.7 months]. The three-year survival rate of patients with high levels of MK (29.8\%) was significantly lower than that of patients with low levels of MK (66.8\%) ( $\mathrm{P}=0.029)$ (Fig. 3A). The median survival time of patients with low expression levels of syndecan-1 was 24 months (95\% CI, 19.7-28.3 months). The three-year survival rate for patients with low expression levels of syndecan-1 (27.3\%) was significantly lower than that for the group with high syndecan-1 levels (53.6\%) ( $\mathrm{P}=0.028$; Fig. 3B).

\section{Discussion}

GCA is a malignant carcinoma that arises in the gastric cardiac tissue. It is one of the most common malignant tumors of the digestive tract in Northern China. The incidence of gastric GCA has increased rapidly (1), whereas that of distal gastric adenocarcinoma has steadily decreased $(2,3)$. Epidemiological and population cohort studies have shown that risk the factors and the clinical and pathological characteristics of GCA are distinctly different from those of gastric cancers that arise in the distal parts, indicating that GCA is a separate disease (15-17). As early symptoms of GCA 
Table IV. Logistic regression analysis for the association between syndecan-1 levels and differentiation, lymph node metastasis or serosal membrane invasion.

95\% CI for $\operatorname{Exp}(\mathrm{B})$

\begin{tabular}{lcccccccc}
\cline { 6 - 9 } Parameter & B & S.E. & Wald & df & P-value & Exp (B) & Lower & Upper \\
\hline Differentiation status & 0.532 & 0.536 & 2.363 & 2 & 0.307 & 1.972 & 0.735 & 5.899 \\
Lymph node metastasis & 0.621 & 0.545 & 1.210 & 1 & 0.271 & 1.821 & 0.626 & 5.299 \\
Serosal membrane invasion & -1.284 & 0.556 & 5.331 & 1 & 0.021 & 0.277 & 0.093 & 0.824 \\
\hline
\end{tabular}

CI, confidence interval; df, degrees of freedom; SE, standard error.

Table V. Correlation between the expression of MK and syndecan- $1{ }^{\mathrm{a}}$

\begin{tabular}{lrrrrr}
\hline & \multicolumn{3}{c}{ MK, $\mathrm{n}$} \\
\cline { 2 - 6 } Syndecan-1 & \multicolumn{1}{c}{} & + & ++ & +++ & Total \\
\hline- & 5 & 9 & 13 & 17 & 44 \\
+ & 2 & 2 & 1 & 3 & 8 \\
++ & 4 & 2 & 2 & 1 & 10 \\
+++ & 6 & 1 & 23 & 10 \\
Total & 17 & 14 & 18 & 23 \\
\hline
\end{tabular}

${ }^{\mathrm{a}} \mathrm{A}$ significant reverse correlation between the expression of MK and syndecan- 1 in GCA tissue $(\mathrm{r}=-0.352, \mathrm{P}<0.01)$ was revealed by Spearman's rank correlation test. MK, midkine; GCA, gastric cardiac adenocarcinoma; (-), <10\% positive cells; (+), 10-49\% positive cells; (++), 50-75\% positive cells; $(+++),>75 \%$ positive cells displaying cytoplasmic and membrane-associated immunoreactivity.

are non-specific and lack sensitive and specific biomarkers for early diagnosis, the majority of patients are diagnosed at advanced stages; therefore, the five-year survival rate for patients with GCA does not exceed $24 \%$, even with comprehensive treatment. Thus, to improve the early diagnosis of GCA, it is important to investigate the molecular mechanisms underlying the development and progression of GCA and to identify possible biomarkers. A number of studies have analyzed the expression of MK or syndecan-1 in distal gastric adenocarcinoma; however, few studies have explored this in GCA (18-20).

Human MK can bind syndecan proteoglycan family members, receptor-type tyrosine kinase- $\zeta$, low-density lipoprotein, receptor-related protein and ALK. MK binds tightly to the syndecan proteins, including syndecan-1, 3 and 4, through HS (9). Deregulation of MK is associated with the pathogenesis of numerous diseases, including cancer $(7,21)$. MK promotes carcinogenesis by enhancing fibrinolysis, cell transformation, migration, cell survival and anti-apoptotic activity through a molecular mechanism that remains to be elucidated (22). The overexpression of MK is common in numerous malignant tumor types and particularly in advanced cancer types, including esophageal $(23)$, gastric $(1,24)$, colorectal (25), liver (26), pancreatic, lung (27) and breast (28) cancer and neuroblastoma (29). The overexpression of MK is closely correlated with tumor development and progression (30); however, expression levels of MK in GCA have yet to be reported.
In the present study, the overexpression of MK was present in 55 out of 72 tumor samples from patients with GCA (76.4\%), which was significantly higher than for normal tissue samples. These results are consistent with the $65-80 \%$ positive rates for gastric cancer $(20,31)$. Furthermore, the MK overexpression rates in the lymph node metastasis and stromal invasion groups were significantly higher than in the groups with no lymph node metastasis $\left(\chi^{2}, 8.5 ; \mathrm{P}<0.05\right)$ and no stromal invasion $\left(\chi^{2}, 5.073 ; \mathrm{P}<0.01\right)$. Logistic regression analysis revealed that high MK expression was associated with lymph node metastases $(\mathrm{P}=0.007)$ and the depth of serosal membrane invasion $(\mathrm{P}=0.018)$. Notably, the increased expression of MK in the tumor tissue was correlated with a shorter median survival time and lower three-year survival rates compared with patients with low MK expression levels. These results indicate that the overexpression of MK in GCA may promote the proliferation of cancer cells and contribute to cancer invasion and metastasis, which is associated with late clinical stages and a poor prognosis for patients with GCA.

Syndecans are transmembrane proteoglycans that carry covalently bound HS side chains. There are four members in this family, syndecan-1, $-2,-3$ and -4 , which are encoded by different genes. Syndecan-1 (CD138) is an important component of the plasma membrane (32); it is a transmembrane HS proteoglycan (HSPG) that is mainly expressed in the epithelial cells. HS is a polysaccharide that usually occurs in the form of HSPG. The HS chains allow for the interaction with a variety of regulatory factors. Syndecan-1 binds to a variety of growth 
factors, including MK, through its HS side chains to regulate cell growth, differentiation, adhesion and migration, as well as cell-cell and cell-extracellular matrix interactions (33). The association between syndecan-1 expression and tumor development is not currently clear. A number of studies have indicated that syndecan-1 inhibits tumor development and is absent or present at extremely low levels in most solid malignant tumors, including head and neck squamous cell, cervical, gastrointestinal and liver cancer $(12,13)$. As syndecan-1 is a cell surface adhesion molecule, complete loss or reduction of syndecan-1 may facilitate the migration of metastatic cells. Therefore, decreased syndecan-1 expression may be indicative of aggressive malignant behavior (34). By contrast, certain studies have demonstrated that syndecan-1 promotes metastasis in rat lung squamous cell cancer (13). Moreover, its expression levels are increased in pancreatic, gastric and breast cancer. Thus, increased expression of syndecan-1 is correlated with tumor invasion, metastasis and a poor prognosis (35).

The results of the present study showed that syndecan-1 was expressed in $100 \%$ of the normal cardiac mucosa tissue samples, whereas only $38.95 \%$ of the GCA samples $\left(\chi^{2}, 40.261\right.$; $\mathrm{P}<0.01)$ were positive for syndecan -1 . These observations indicate that a loss of syndecan-1 expression is associated with the development of GCA. The expression levels of syndecan-1 were associated with the degree of differentiation. A total of 72.7, 35.6 and $25 \%$ of cells expressed syndecan- 1 in the samples with high, medium and low degrees of differentiation, respectively. Statistical analysis revealed that low expression levels of syndecan-1 were significantly correlated with the degree of differentiation, presence of lymph node metastases, stromal invasion of GCA, short median survival time and low three-year survival rate $(\mathrm{P}<0.01)$. Logistic regression analysis showed that syndecan-1 expression was correlated only with serosal membrane invasion. These results indicate that syndecan-1 expression may be a prognostic marker for patients with GCA. However, other studies have indicated that patients with gastric cancer with low epithelial syndecan-1 expression levels have poor overall survival rates (36). In addition, high stromal syndecan-1 expression levels have been shown to correlate with decreased epithelial syndecan-1 expression, which led to significantly reduced survival times in females (36). Thus, further evaluation of the expression levels of epithelial and stromal syndecan-1 in GCA tissue is required to ascertain its prognostic value.

Furthermore, the statistical analysis performed in the present study showed a strong inverse correlation between MK and syndecan-1 ( $\mathrm{r}=-0.352, \mathrm{P}<0.01)$. The overexpression of MK was frequently detected in GCA tissue in which syndecan-1 was absent, indicating that MK and syndecan-1 are involved in the development and progression of GCA. The concomitant loss of syndecan-1 and overexpression of $\mathrm{MK}$ may promote the development of GCA. Loss of syndecan-1 on the tumor cell surface may be due to increased enzymatic activity of heparanase, which degrades the extracellular HS chains of syndecan-1. This loss of HS chains may negatively affect the interaction of syndecan-1 with heparin-binding growth factors, including fibroblast growth factor (FGF), hepatocyte growth factor and vascular endothelial growth factor (37). Numerous studies have demonstrated that basic
FGF (bFGF) is inactive when HS is present on the cell surface membrane or in the extracellular matrix, but its biological activity is restored when it is released from the cell surface following hydrolysis of HS by acetyl-heparanase $(38,39)$.

The mechanism by which syndecan-1 and MK impact the development of GCA remains to be elucidated. Although the statistical analysis performed in this study showed that there is a correlation between the expression of MK and syndecan-1 in GCA tissue, the functional link between these two molecules may be affected by interactions with other factors, as MK binds to a wide variety of receptors. The interaction with these receptors may exert a synergistic affect on the biological activity of MK. Thus, further studies are required to enhance our understanding of the mechanisms by which MK and syndecan-1 interact with each other.

\section{Acknowledgements}

This study was supported by the National Natural Science Foundation of China (no. U1204819).

\section{References}

1. Wang LD, Qin YR, Fan ZM, et al: Comparative genomic hybridization: comparison between esophageal squamous cell carcinoma and gastric cardia adenocarcinoma from a high-incidence area for both cancers in Henan, northern China. Dis Esophagus 19: 459-467, 2006.

2. Devesa SS, Blot WJ and Fraumeni JF Jr: Changing patterns in the incidence of esophageal and gastric carcinoma in the United States. Cancer 83: 2049-2053, 1998.

3. Botterweck AA, Schouten LJ, Volovics A, Dorant E and van Den Brandt PA: Trends in incidence of adenocarcinoma of the oesophagus and gastric cardia in ten European countries. Int J Epidemiol 29: 645-654, 2000.

4. Kadomatsu K and Muramatsu T: Midkine and pleiotrophin in neural development and cancer. Cancer Lett 204: 127-143, 2004.

5. Zhao ZQ, Yang S and Lu HS: Expression of midkine and vascular endothelial growth factor in gastric cancer and the association of high levels with poor prognosis and survival. Mol Med Rep 5: 415-419, 2012.

6. Huang Y, Cao G, Wang H, Wang Q and Hou Y: The expression and location of midkine in gastric carcinomas of Chinese patients. Cell Mol Immunol 4: 135-140, 2007.

7. Muramatsu T: Midkine, a heparin-binding cytokine with multiple roles in development, repair and diseases. Proc Jpn Acad Ser B Phys Biol Sci 86: 410-425, 2010.

8. Muramatsu T: Midkine and pleiotrophin: two related proteins involved in development, survival, inflammation and tumorigenesis. J Biochem 132: 359-371, 2002.

9. Mitsiadis TA, Salmivirta M, Muramatsu T, et al: Expression of the heparin-binding cytokines, midkine (MK) and HB-GAM (pleiotrophin) is associated with epithelial-mesenchymal interactions during fetal development and organogenesis. Development 121: 37-51, 1995.

10. Bernfield M, Kokenyesi R, Kato M, et al: Biology of the syndecans: a family of transmembrane heparan sulfate proteoglycans. Annu Rev Cell Biol 8: 365-393, 1992.

11. Teng YH, Aquino RS and Park PW: Molecular functions of syndecan-1 in disease. Matrix Biol 31: 3-16, 2012.

12. Anttonen A, Kajanti M, Heikkilä P, Jalkanen M and Joensuu $\mathrm{H}$ : Syndecan-1 expression has prognostic significance in head and neck carcinoma. Br J Cancer 79: 558-564, 1999.

13. Hirabayashi K, Numa F, Suminami Y, et al: Altered proliferative and metastatic potential associated with increased expression of syndecan-1. Tumour Biol 19: 454-463, 1998.

14. Harada K, Masuda S, Hirano M and Nakanuma Y: Reduced expression of syndecan-1 correlates with histologic dedifferentiation, lymph node metastasis, and poor prognosis in intrahepatic cholangiocarcinoma. Hum Pathol 34: 857-863, 2003. 
15. Wu X, Chen VW, Andrews PA, Ruiz B and Correa P: Incidence of esophageal and gastric cancers among Hispanics, non-Hispanic whites and non-Hispanic blacks in the United States: subsite and histology differences. Cancer Causes Control 18: 585-593, 2007.

16. Wijetunge S, Ma Y, DeMeester S, et al: Association of adenocarcinomas of the distal esophagus, 'gastroesophageal junction,' and 'gastric cardia' with gastric pathology. Am J Surg Pathol 34: $1521-1527,2010$

17. Xiao ZY, Ru Y, Sun JT, et al: Expression of CDX2 and villin in gastric cardiac intestinal metaplasia and the relation with gastric cardiac carcinogenesis. Asian Pac J Cancer Prev 13: 247-250, 2012.

18. Ding GC, Ren JL, Chang FB, et al: Human papillomavirus DNA and P16(INK4A) expression in concurrent esophageal and gastric cardia cancers. World J Gastroenterol 16: 5901-5906, 2010.

19. Chu YQ, Ye ZY, Tao HQ, et al: Relationship between cell adhesion molecules expression and the biological behavior of gastric carcinoma. World J Gastroenterol 14: 1990-1996, 2008.

20. Obata Y, Kikuchi S, Lin Y, et al: Serum midkine concentrations and gastric cancer. Cancer Sci 96: 54-56, 2005.

21. Sakamoto K and Kadomatsu K: Midkine in the pathology of cancer, neural disease, and inflammation. Pathol Int 62: 445-455, 2012.

22. Michikawa M, Kikuchi S, Muramatsu H, Muramatsu T and Kim SU: Retinoic acid responsive gene product, midkine, has neurotrophic functions for mouse spinal cord and dorsal roo ganglion neurons in culture. J Neurosci Res 35: 530-539, 1993.

23. Shimada H, Nabeya Y, Okazumi S, et al: Increased serum midkine concentration as a possible tumor marker in patients with superficial esophageal cancer. Oncol Rep 10: 411-414, 2003

24. Xu YY, Mao XY, Song YX, et al: Midkine confers Adriamycin resistance in human gastric cancer cells. Tumour Biol 33 1543-1548, 2012.

25. Krzystek-Korpacka M, Diakowska D, Grabowski K and Gamian A: Tumor location determines midkine level and its association with the disease progression in colorectal cancer patients: a pilot study. Int J Colorectal Dis 27: 1319-1324, 2012.

26. Hung YJ, Lin ZH, Cheng TI, Liang CT, Kuo TM and Kao KJ: Serum midkine as a prognostic biomarker for patients with hepatocellular carcinoma. Am J Clin Pathol 136: 594-603, 2011.
27. Zhao G, Nie Y, Lv M, He L, Wang T and Hou Y: ER $\beta$-mediated estradiol enhances epithelial mesenchymal transition of lung adenocarcinoma through increasing transcription of midkine. Mol Endocrinol 26: 1304-1315, 2012.

28. Ibusuki M, Fujimori H, Yamamoto Y, et al: Midkine in plasma as a novel breast cancer marker. Cancer Sci 100: 1735-1739, 2009.

29. Reiff T, Huber L, Kramer M, Delattre O, Janoueix-Lerosey I and Rohrer $\mathrm{H}$ : Midkine and Alk signaling in sympathetic neuron proliferation and neuroblastoma predisposition. Development 138: 4699-4708, 2011

30. Fabri L, Maruta H, Muramatsu H, et al: Structural characterisation of native and recombinant forms of the neurotrophic cytokine MK. J Chromatogr 646: 213-225, 1993.

31. Zhao ZQ, Yang S, Lu HS, et al: Expression of midkine and vascular endothelial growth factor in gastric cancer and the association of high levels with poor prognosis and survival. Mol Med Rep 5: 415-419, 2012.

32. Teng YH, Aquino RS and Park PW: Molecular functions of syndecan-1 in disease. Matrix Biol 31: 3-16, 2012.

33. Fux L, Ilan N, Sanderson RD and Vlodavsky I: Heparanase: busy at the cell surface. Trends Biochem Sci 34: 511-519, 2009.

34. Huang MF, Zhu YQ, Chen ZF, et al: Syndecan-1 and E-cadherin expression in differentiated type of early gastric cancer. World J Gastroenterol 11: 2975-2980, 2005.

35. Conejo JR, Kleeff J, Koliopanos A, et al: Syndecan-1 expression is up-regulated in pancreatic but not in other gastrointestinal cancers. Int J Cancer 88: 12-20, 2000.

36. Wiksten JP, Lundin J, Nordling S, et al: Epithelial and stromal syndecan-1 expression as predictor of outcome in patients with gastric cancer. Int J Cancer 95: 1-6, 2001.

37. Vlodavsky I, Friedmann Y, Elkin M, et al: Mammalian heparanase: gene cloning, expression and function in tumor progression and metastasis. Nat Med 5: 793-802, 1999.

38. Naggi A, Casu B, Perez M, et al: Modulation of the heparanase-inhibiting activity of heparin through selective desulfation, graded N-acetylation, and glycol splitting. J Biol Chem 280: 12103-12113, 2005.

39. Kato M, Wang H, Kainulainen V, et al: Physiological degradation converts the soluble syndecan-1 ectodomain from an inhibitor to a potent activator of FGF-2. Nat Med 4: 691-707, 1998. 